\title{
Inducible Lytic Systems in the Genus Bacillus
}

\author{
By R. A. ALTENBERN and H. B. STULL \\ U.S. Army Biological Laboratories, Fort Detrick, \\ Frederick, Maryland, U.S.A.
}

(Received 12 October 1964)

\begin{abstract}
SUMMARY
Many different strains of Bacillus cereus and members of other species of the genus Bacillus were induced to lyse by exposure to the proper concentration of mitomycin $\mathrm{C}$, a radiomimetic drug. For some bacilli, induction of lysis occurred only in Casamino acids containing medium but not in nutrient broth + yeast extract medium, whereas other bacilli showed the opposite behaviour. The efficiency of induction was a function of the growth phase of the culture and of the concentration of mitomycin C. Induction of lysis in B. thuringiensis resulted in the release of oedema factor and phospholipase into the medium. Mitomycin $\mathrm{C}$-induced lysis of $\boldsymbol{B}$. cereus was prevented by adding chloramphenicol, actinomycin $\mathrm{D}$, or 5 -fluorouracil deoxyriboside up to $40 \mathrm{~min}$. later. There appeared to be no sequential loss of susceptibility to inhibition by these compounds as expected.
\end{abstract}

\section{INTRODUCTION}

The close relationship between lysis induced by ultraviolet (u.v.) radiation and the production of the oedema-producing toxin by a strain of Bacillus cereus (6464) was reported by Altenbern (1962). Further work showed that either u.v.-radiation or mitomycin $\mathrm{C}$, a radiomimetic agent, could be used as an inducing agent. Many other strains of $\boldsymbol{B}$. cereus tested gave mitomycin $\mathrm{C}$-induced lysis like that observed for strain 6464. The study was then expanded to include many species of the genus Bacillus and sometimes several strains of a species. The present paper is mainly a survey of the action of mitomycin $\mathrm{C}$ on members of the genus Bacillus, including the effect of medium on ease of induction, the rate of induced lysis, the production of phospholipase and toxin by strains of $B$. thuringiensis and $\boldsymbol{B}$. anthracis, and the effects of chloramphenicol, actinomycin D and 5-fluorouracil deoxyriboside on the course of mitomycin $\mathrm{C}$-induced lysis in certain species.

\section{METHODS}

The various organisms used in this work were maintained as spore stocks in soil. Regular spore suspensions were prepared from potato-extract agar slopes as described by Thorne (1962). Two media were routinely used: (1) the Casamino acids medium; (2) the nutrient broth + yeast extract-medium (NBY) described by Thorne (1962).

Cultures of the various organisms in logarithmic growth were prepared by inoculation of tubes with $5 \mathrm{ml}$. of either of the liquid media with enough spores to give finally $10^{6} \mathrm{spores} / \mathrm{ml}$. These tubes were then incubated on a shaker at $37^{\circ}$ until the 
extinction (Coleman, Model 14) at $650 \mathrm{~m} \mu\left(E_{650}\right)$ attained a value of 0.200-0.250. At this time mitomycin $\mathrm{C}$ was added to the desired final concentration, the extinction recorded and the tubes returned to the shaker. Extinction readings were taken at $30 \mathrm{~min}$. intervals until the end of the experiment. On occasion, replicate tubes were prepared and at $\mathbf{3 0} \mathrm{min}$. intervals one culture was filtered through a sintered glass filter and the filtrate assayed for toxin (oedema factor) and phospholipase by the methods described by Molnar (1962).

Stock solutions of mitomycin C $(0.1 \mathrm{mg} . / \mathrm{ml}$.), chloramphenicol (1 mg./ml.), actinomycin $\mathrm{D}$ (1 mg./ml.), and 5-fluorouracil deoxyriboside (5-FUDR; $1 \mathrm{mg} . / \mathrm{ml}$.) were prepared in $0 \cdot 1 \mathrm{M}$-phosphate buffer $(\mathrm{pH} \% \cdot 4)$ and sterilized by filtration through a sintered glass filter. These solutions were stored at $4^{\circ}$ until used.

\section{RESULTS}

\section{Effect of concentration of mitomycin $C$ on induction of lysis of Bacillus cereus strains 6464 and 317}

Logarithmically growing cultures of the two strains in Casamino acids medium received additions of mitomycin $\mathrm{C}$ in a range from 0.1 to $1.0 \mu \mathrm{g} . / \mathrm{ml}$. Extinction measurements were made as described under Methods and the results are presented in Fig. 1. It is clear that under these conditions $0 \cdot 1-0.5 \mu \mathrm{g}$. mitomycin $\mathrm{C} / \mathrm{ml}$. caused maximum lysis, and both growth and lysis were prevented by $1.0 \mu \mathrm{g} . / \mathrm{ml}$. There was a rather critical concentration of organism equivalent to an extinction of $E_{650}$, 0.300 at which the mitomycin $\mathrm{C}$ was an effective inducer of lysis, but above this value, little induction by mitomycin $\mathrm{C}$ was obtained. Mitomycin $\mathrm{C}$ induction of $B$. cereus strain 6464 resulted in a pronounced release of mature phage particles and seemed to be at least as effective as u.v.-radiation as an inducing agent.

\section{Inducibility of Bacillus cereus and other species of Bacillus}

Many strains of Bacillus cereus and other species of the genus were examined for lysis caused by mitomycin C. B. cereus strains w, 7004, 7064, 9139 and B-569 were all readily inducible by mitomycin $\mathrm{C}$ as they were with u.v.-radiation. Figure 2 gives some results obtained with four other species (B. subtilis, brevis, vulgatus, thuringiensis no. 98) for comparison with $B$. cereus strain 6464 . In each case, there seemed to be an individual response of the organism to mitomycin $C$, in that for some organisms relatively high concentrations of mitomycin $\mathrm{C}(1.0 \mu \mathrm{g} . / \mathrm{ml}$.$) pro-$ duced some lysis (B. subtilis, B. brevis), whereas with $B$. thuringiensis this concentration of mitomycin $C$ prevented lysis. Other dissimilarities such as the time at which lysis began as a function of concentration of inducer and the rate of lysis once initiated, are difficult to interpret.

\section{The effect of composition of the culture medium on inducibility}

Reich, Shatkin \& Tatum (1961) reported that the medium exerted considerable influence on the sensitivity to mitomycin $\mathrm{C}$ of lysogenic and non-lysogenic bacteria. We found that some species of Bacillus show excellent mitomycin C-induced lysis in the Casamino acids medium but not in the nutrient broth + yeast-extract medium, and other species showed the opposite effect. In Fig. 3 are presented results 
obtained with $B$. subtilis and B. anthracis Sterne strain, to illustrate this behaviour. Also B. brevis, B. vulgatus and B. licheniformis showed good lysis in the Casamino acids medium but not in the nutrient broth + yeast-extract medium, in contrast to $B$. anthracis Sterne strain and B. cereus strain 7004, which gave good lysis in nutrient broth + yeast-extract medium but not in the Casamino acids medium. Reports of

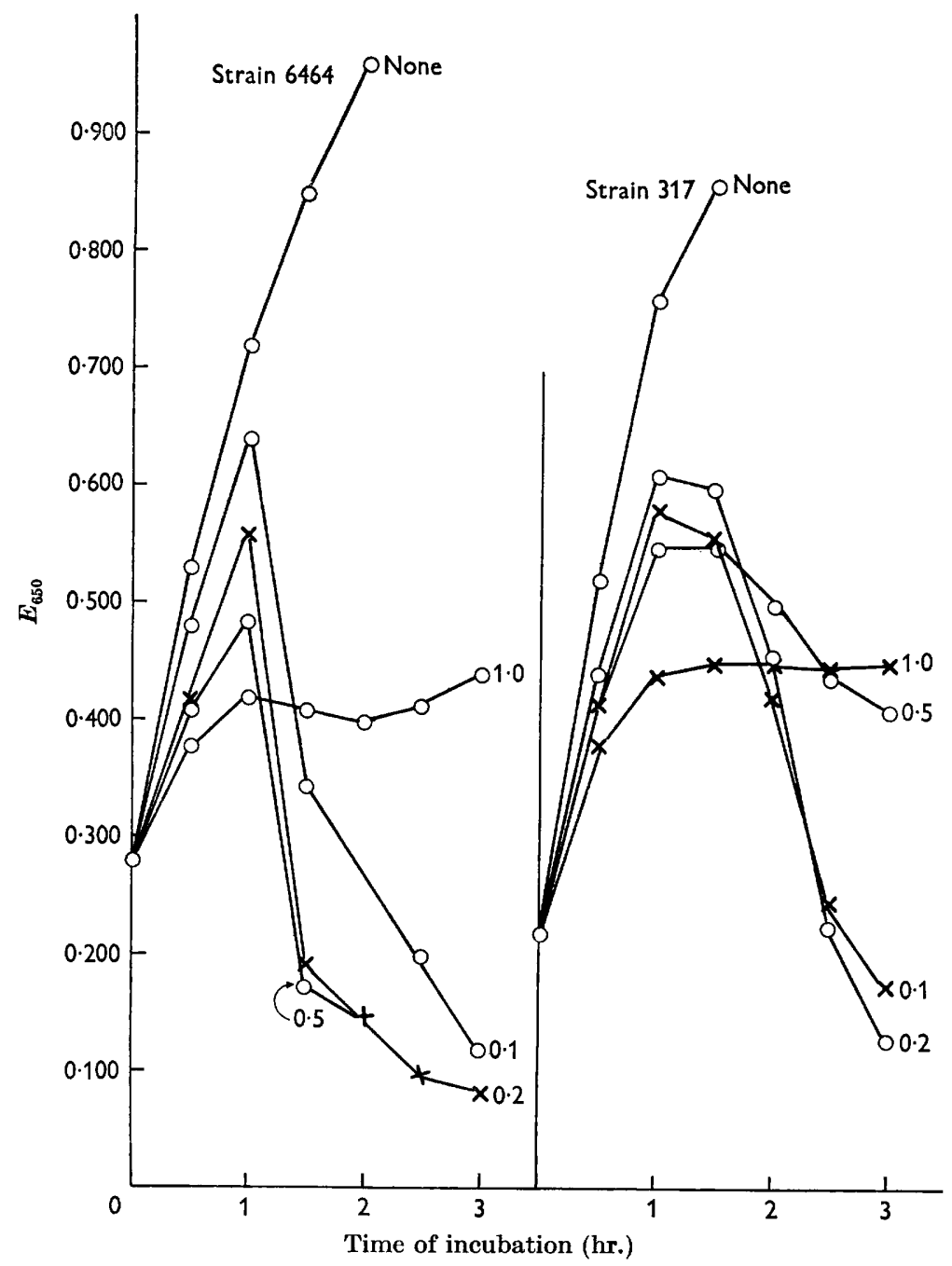

Fig. 1. The induction of lysis of two strains of Bacillus cereus by mitomycin C. Organisms grown in Casamino acids medium. Numbers attached to curves refer to mitomycin $\mathrm{C}$ $\mu \mathrm{g} . / \mathrm{ml}$.

such sensitivity of the lytic response to the composition of the culture medium are not common. Faguet (1962) showed the marked effect of adenosine triphosphate or glycerol on the lysis of induced lysogenic Escherichia coli. In contrast to the strains of $B$. cereus, induced lysis of $B$. anthracis Sterne strain in nutrient broth + yeast extract medium, as noted above, did not release large amounts of phospholipase, 
oedema factor or protective antigen into the medium. However, mitomycin Cinduced lysis of $\boldsymbol{B}$. thuringiensis was accompanied by release of oedema factor and phospholipase into the medium (see Table 1). In this regard, B. thuringiensis resembles the various strains of $B$. cereus, yet it is of interest since spores of this organism are used rather indiscriminately in insect control in the U.S.A.

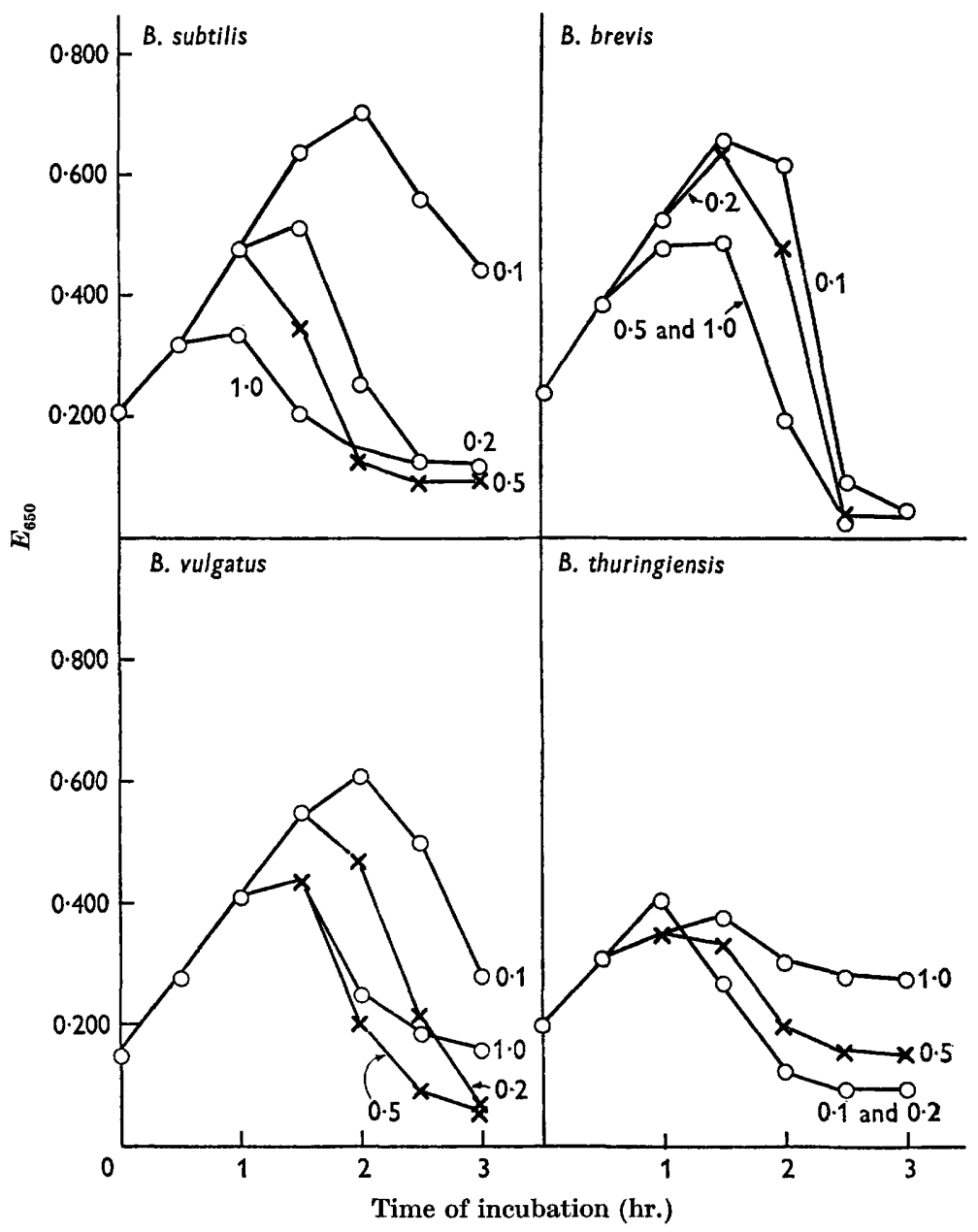

Fig. 2. The induction of four species of Bacillus with varying amounts of mitomycin $\mathbf{C}$. Strains grown in Casamino acids medium. Numbers attached to curves refer to mitomycin $\mathrm{C} \mu \mathrm{g} . / \mathrm{ml}$.

\section{Action of inhibitors of macromolecular syntheses on mitomycin $C$-induced lysis}

To examine the nature of lysis caused by mitomycin $\mathrm{C}$, the effect of various inhibitors on induced lysis was determined. Chloramphenicol, an inhibitor of protein synthesis, was a potent inhibitor of the mitomycin C-induced lysis of many species of Bacillus. Figure 4 shows results obtained with four species of Bacillus, and illustrates the prevention of induced lysis. Other experiments with $B$. cereus strains 6464 and 317 showed that chloramphenicol inhibited lysis when added 


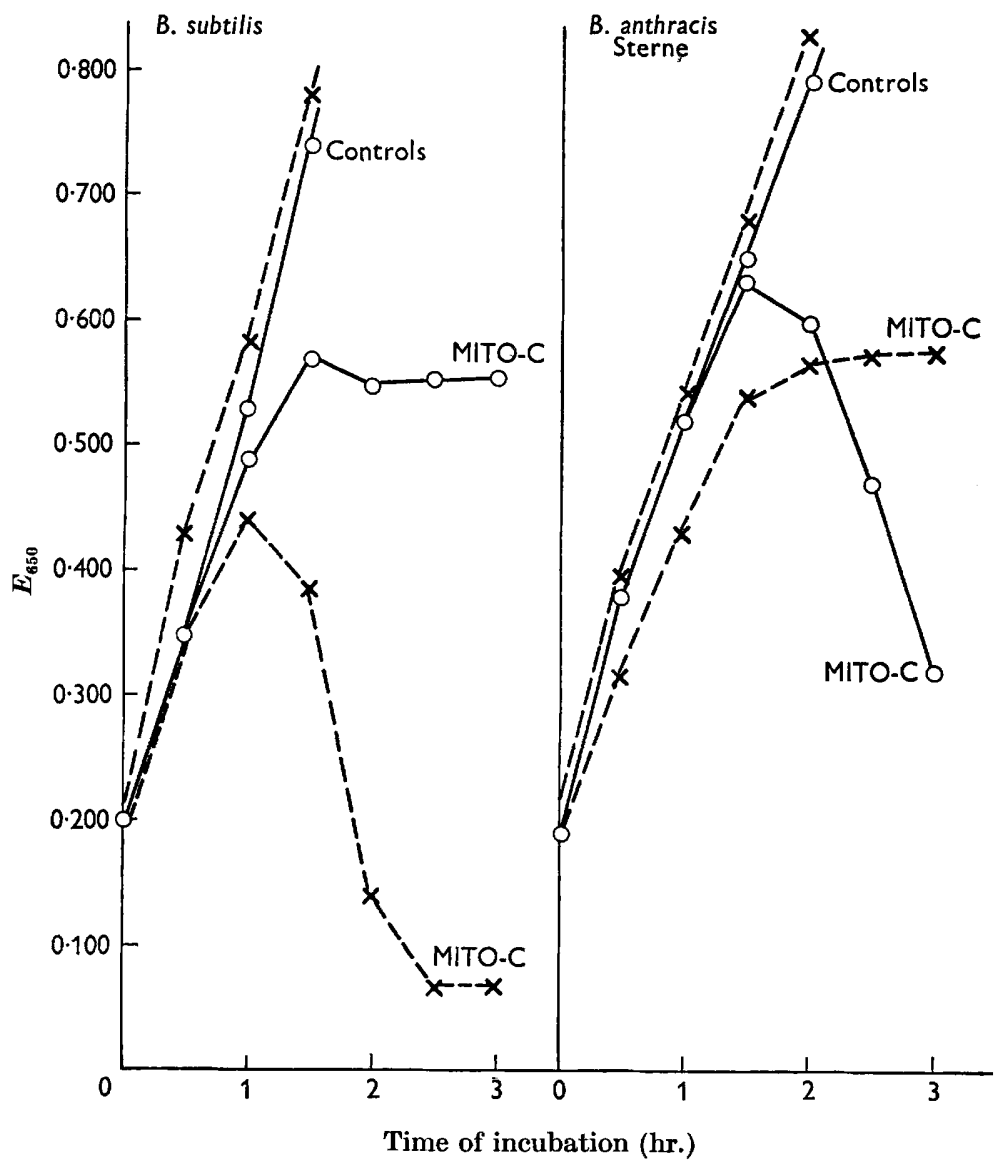

Fig. 3. Induction by mitomycin $\mathrm{C}$ of two species of Bacillus as influenced by growth medium. All inductions performed with mitomycin $\mathrm{C}$ in a final concentration of $0.2 \mu \mathrm{g} . / \mathrm{ml}$. $\bigcirc-\bigcirc$, Nutrient broth + yeast extract medium; $\times \cdots \times$, Casamino acids medium.

Table 1. Production of phospholipase and toxin during mitomycin $C$ induced lysis of Bacillus thuringiensis

Mitomycin C addition: $\mathbf{0 . 2} \mu \mathrm{g} . / \mathrm{ml}$. Organism grown in nutrient broth + yeast-extract medium.

Time after adding mitomycin $\mathbf{C}$

Phospho(min.) (units/ml.)

Toxin

(units $/ \mathrm{ml}$.)

2

4

32

16

64

64
$E_{650}$

$0 \cdot 240$

$0 \cdot 470$

0.565

$\mathbf{0 . 5 8 0}$

0.500

0.420

$0 \cdot 340$

$0 \cdot 290$ 
20 min. after adding mitomycin $\mathrm{C}$ but not after $40 \mathrm{~min}$. (Fig. 5). However, the time required to yield a detectable degree of lysis when chloramphenicol was added 40 min. after adding mitomycin $\mathrm{C}$ was considerably extended and indicates that the comparatively lesser amount of protein produced (lytic enzyme?) took longer to

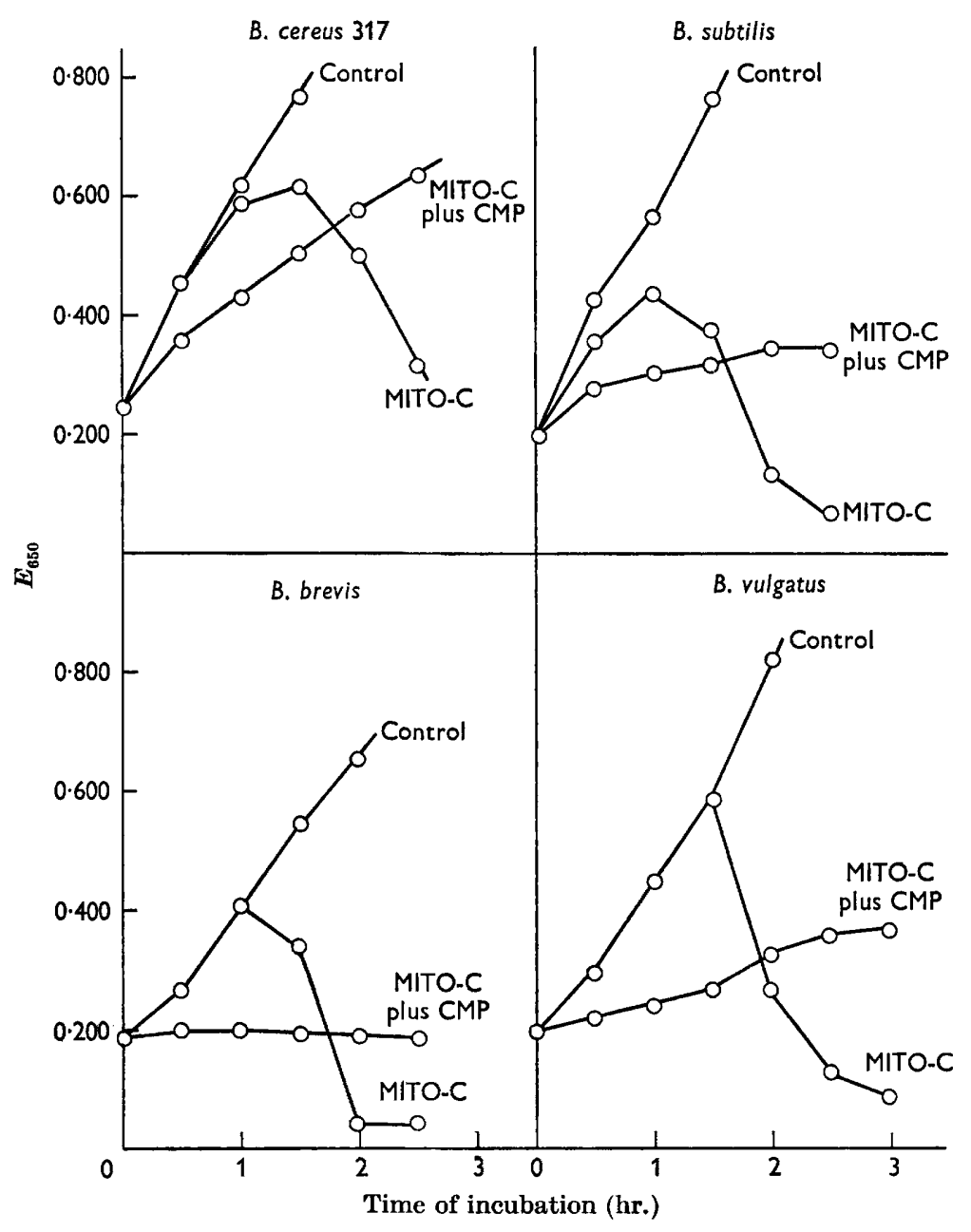

Fig. 4. The effect of chloramphenicol (CMP) on mitomycin $\mathrm{C}$ induction of four species of Bacillus. All inductions performed with mitomycin C $0 \cdot 2 \mu \mathrm{g} . / \mathrm{ml}$. Chloramphenicol added to $10 \mu \mathrm{g}$. $/ \mathrm{ml}$. final concentration at time of adding mitomycin $\mathrm{C}$.

alter the bacilli so that lysis ensued. The rate at which lysis proceeds seems to be independent of the time required for lysis to begin, suggesting that the lytic event per $s e$ is non-enzymic.

Further work showed that 5-fluorouracil deoxyriboside (5-FUDR) and actinomycin D were potent inhibitors of mitomycin C-induced lysis in Bacillus cereus strains 6464 and 317; the data are presented in Fig. 6. Further study of the effect of the time of addition of 5-FUDR or actinomycin D on inhibition of mitomycin Cinduced lysis was made. As with chloramphenicol, lysis was prevented by the 
addition of 5-FUDR or actinomycin $\mathrm{D}$ at any time up to $40 \mathrm{~min}$. after adding mitomycin C (Fig. 6). There was no evidence that the induced lytic system lost sensitivity to an inhibitor of DNA synthesis early, or that inhibition of lysis by chloramphenicol could be effected for a significantly longer time than the inhibition by the two other inhibitors.

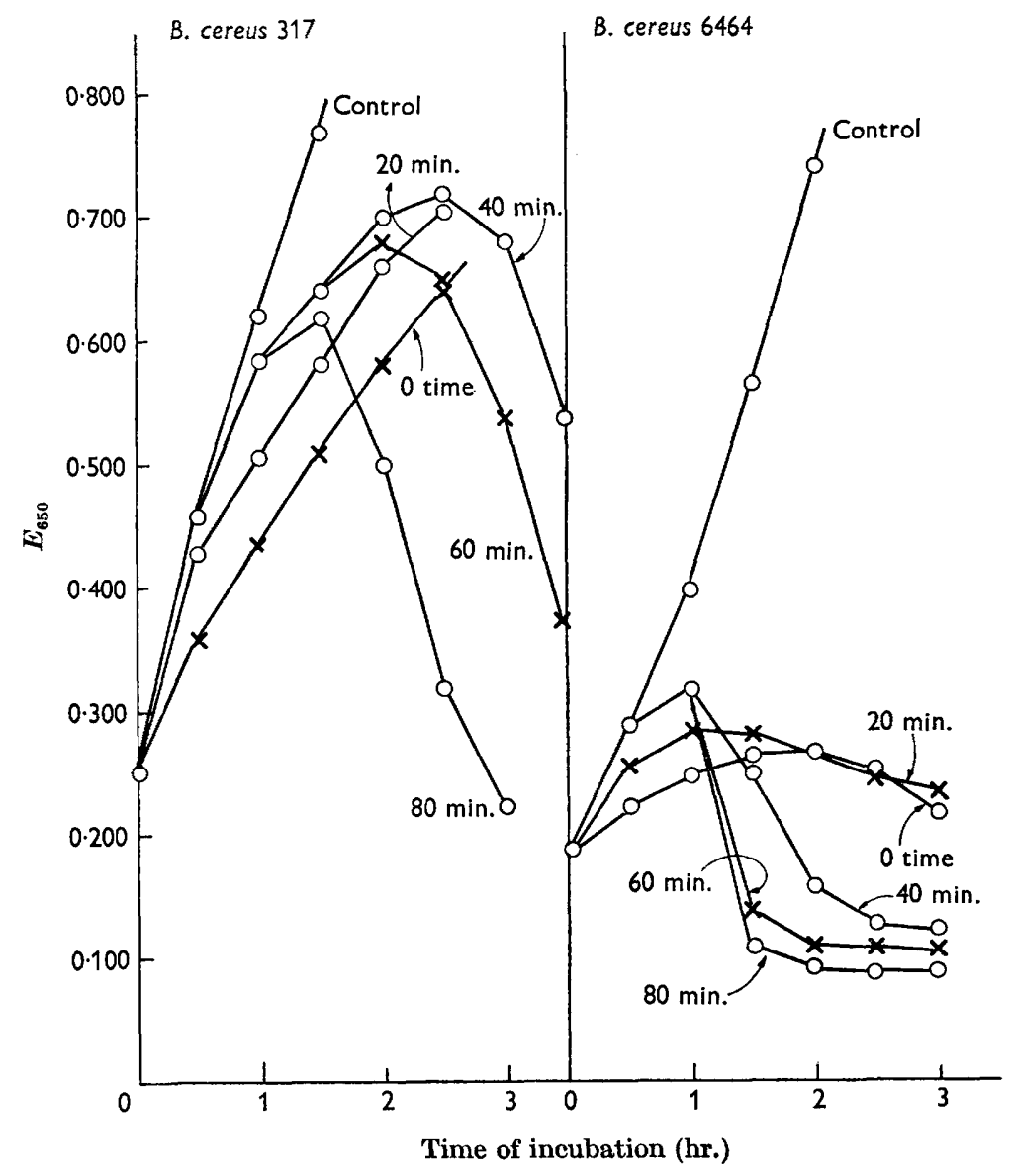

Fig. 5. The effect on the lysis induced by mitomycin $\mathrm{C}$ of adding chloramphenicol. All inductions performed with mitomycin $\mathrm{C} 0 \cdot 2 \mu \mathrm{g}$. $/ \mathrm{ml}$. Chloramphenicol added to $10 \mu \mathrm{g} . / \mathrm{ml}$. final concentration at the times indicated. Organisms were grown in Casamino acids medium.

Two other substituted deoxyuridines, 5-bromouracil deoxyriboside and 5-iodouracil deoxyriboside, did not prevent mitomycin $\mathrm{C}$-induced lysis of Bacillus cereus strains 6464 and 317 even when these compounds were added to the cultures 20 min. before adding mitomycin C. 5-Fluorouracil deoxyriboside prevented mitomycin C-induced lysis in $B$. globigii grown in Casamino acids medium, but 5-FUDR did not inhibit the induced lysis of $B$. brevis, B. agri or B. vulgatus, all when growing in the nutrient broth + yeast extract medium. The possible effect here of the composition of the medium on the action of this inhibitor was not further investigated. 


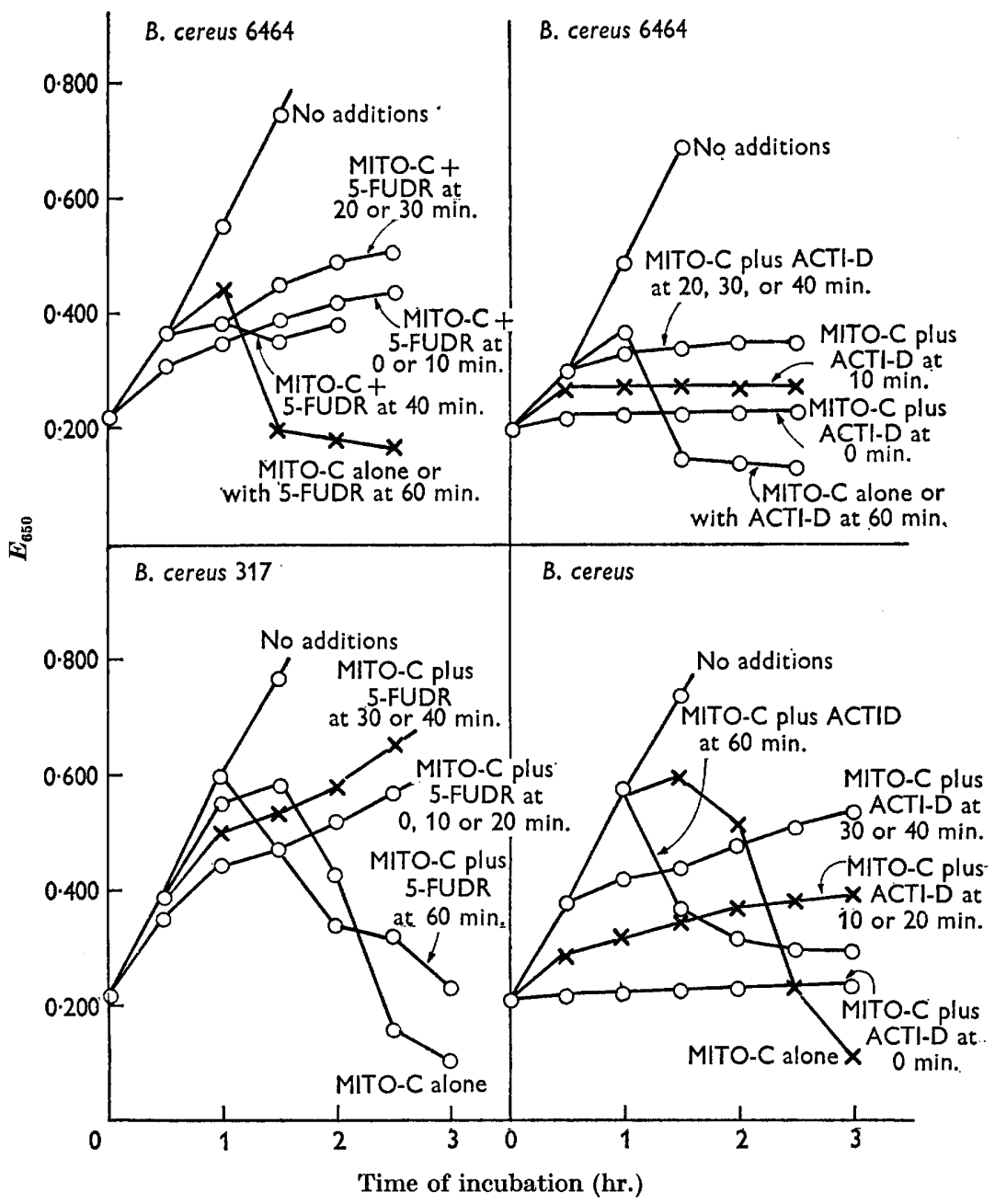

Fig. 6. The effect of addition of 5-fluorouracil deoxyriboside (5-FUDR) or actinomycin $D$ (acti-D) on the lysis induced by mitomycin C. All induction with mitomycin C $0.2 \mu \mathrm{g} . / \mathrm{ml}$. 5-FUDR (10 $\mu \mathrm{g} . / \mathrm{ml}$.) or actinomycin D $(0.5 \mu \mathrm{g} . / \mathrm{ml}$.$) added after induction as indicated.$ Organisms were grown in Casamino acids medium.

\section{DISCUSSION}

Induction of lysogenic bacteria with ultraviolet radiation or with mitomycin $\mathrm{C}$ is well known. Induction of lysogenic Escherichia coli by mitomycin $\mathrm{C}$ was reported by Otsugi, Sekiguchi, Iijima \& Takagi (1959) who showed that a small concentration $(0 \cdot 1-1 \cdot 0 \mu \mathrm{g} . / \mathrm{ml}$.) effected pronounced lysis, whereas larger concentrations $(5 \cdot 0 \mu \mathrm{g} . /$ ml.) caused growth inhibition without inducing lysis. All the strains of the various Bacillus species examined in the present work exhibited such a response, although only one strain (6464) of Bacillus cereus possessed a complete prophage which replicated after induction and produced plaque-forming units. Induction of lysis by a radiomimetic agent such as mitomycin $\mathrm{C}$ may offer presumptive evidence of 
lysogeny even in the absence of a suitable indicator strain to detect free phage. In addition, some types of defective lysogeny might be detected by this technique, whereas plating on selected indicator strains would invariably yield negative results. Electron microscopy of sedimentable material from lysates of these organisms was not used but might reveal defective but morphologically distinct phage particles. In our work no effort was made to detect mature bacteriophage particles in lysates of these cultures by plating filtrates on potential indicator strains. Csuzi \& Kramer (1962) showed that there is an u.v.-radiation-inducible lytic system in $B$. cereus strain B-569, and showed by determination of soluble protein that measurement of the extinction of cultures was a valid assessment of the extent and rate of lysis.

The results obtained with inhibitors give indications of the mechanism of mitomycin $\mathrm{C}$-induced lysis. It was assumed that mitomycin $\mathrm{C}$ induced a prophage which then directed, through RNA, the synthesis of an enzyme which would lyse the cell. It followed that an inhibitor of DNA synthesis should block replication of the detached (induced) prophage and thus prevent post-induction lysis. Similarly, an inhibitor of RNA synthesis would prevent the formation of new messenger RNA and should thus block synthesis of the lytic enzyme. Finally, chloramphenicol should prevent post-induction lysis by blocking protein (enzyme) synthesis. However, it seemed that there should be a demonstrable sequence of inhibition wherein susceptibility to inhibitors of DNA synthesis should disappear within 10-15 min. after induction, while inhibition by inhibitors of RNA synthesis would be effected 20-25 min. after induction and inhibitors of protein synthesis should prevent lysis when added very late after induction. In contrast to expectation, however, no such sequence of inhibitions was observed and the susceptibility of induced lysis to any of the inhibitors tested seemed to disappear after $40 \mathrm{~min}$. of post-induction. However, DNA, RNA and protein all appear to be involved in the lysis which follows addition of mitomycin $\mathrm{C}$.

Seaman, Tarmy \& Marmur (1964) showed that Bacillus subtilis 168 possessed an inducible prophage but found no replication of the phage DNA and thus the inducible lysis was not prevented by 5-FUDR, an inhibitor of DNA synthesis. We found that mitomycin $\mathrm{C}$-induced lysis of some Bacillus species was prevented by 5-FUDR, although other strains lysed following exposure to mitomycin $\mathrm{C}$ even in the presence of 5-FUDR. It is possible that those cultures in which 5-FUDR had no effect on mitomycin $\mathrm{C}$-induced lysis possessed an inducible but non-replicating prophage as in the case cited above, whereas cultures whose induced lysis was prevented by 5 -FUDR possessed an inducible and replicating prophage. Why replication of a prophage is necessary to produce lysis in some cases but not in others is a matter of conjecture. Although our speculation about the nature of the inducible lysis reported here has emphasized the possible involvement of lysogeny, it is recognized that equally plausible interpretations not involving lysogeny may be made.

The authors wish to thank Hoffman-LaRoche, Inc. for the gift of a supply of 5-fluorouracil deoxyriboside and Merck, Sharp \& Dohme, Inc. for the generous gift of a sample of actinomycin $\mathbf{D}$.

In the work reported here, the investigators adhered to 'Principles of Laboratory Animal Care' as established by the National Society for Medical Research (U.S.A.). 


\section{REFERENCES}

Altenbern, R. A. (1962). Lysogeny and toxinogeny in Bacillus cereus. Biochem. Biophys. Res. Communs. 9, 109.

Csuzi, S. \& Kramer, M. (1962). Production of a lytic factor by ultraviolet light irradiated cultures of Bacillus cereus. Acta microbiol. hung. 9, 297.

FAGUET, M. (1962). Étude de l'influence inhibitrice de certains composes chimiques sur l'induction de la lyse et la production de phages provoquées par l'irradiation de cultures d'Escherichia coli lysogène par un rayonnement ultraviolet. C. r. hebd. Seanc. Acad. Sci., Paris, 255, 1810.

Molvar, D. M. (1962). Separation of the toxin of Bacillus cereus into two components and non-identity of the toxin with phospholipase. J. Bact. 84, 147.

Otsugr, N., Sekrguchi, M., IIJIma, T. \& TAKagi, Y. (1959). Induction of phage formation in the lysogenic Escherichia coli K-12 by mitomycin C. Nature, Lond., 184, 1079.

Reich, E., Shatkin, A. J. \& Tatum, E. L. (1961). Bacteriocidal action of mitomycin C. Biochim. biophys. Acta, 53, 132.

Seaman, E., Tarmy, E. \& Marmur, J. (1964). Inducible phages of Bacillus subtilis. Biochemistry, 3, 607.

ThоRNE, C. B. (1962). Transduction in Bacillus subtilis. J. Bact. 83, 106. 